SI-HEP-2009-15

\title{
HADRONIC FORM FACTORS: COMBINING QCD CALCULATIONS WITH ANALYTICITY*
}

\author{
A. KHODJAMIRIAN \\ Theoretische Physik 1, Fachbereich Physik, Universität Siegen, \\ D-57068 Siegen, Germany \\ E-mail: khodjam@hep.physik.uni-siegen.de
}

\begin{abstract}
I discuss recent applications of QCD light-cone sum rules to various form factors of pseudoscalar mesons. In this approach both soft and hard contributions to the form factors are taken into account. Combining QCD calculation with the analyticity of the form factors, one enlarges the region of accessible momentum transfers.
\end{abstract}

\section{Introduction}

At the beginning of this talk let me quote Misha Shifman:

"Unlike some models whose relation to Nature is still a big question mark, Quantum Chromodynamics will stay with us forever." ${ }^{1}$

In the strong coupling domain, the quark-gluon gauge dynamics of QCD manifests itself in a form of hadrons. A comprehensive analytic description of hadrons and their interactions in QCD remains an unsolved problem that may also stay with us forever. Today we have at our disposal only approximate methods and effective theories of hadrons, with an impressive progress achieved in the numerical simulation of QCD on the lattice.

An approximate analytical calculation of hadronic observables has been made possible with the advent of QCD sum rules. ${ }^{2}$ Not only the original SVZ method is still being extensively used, but also its "offspring", the light-cone sum rules (LCSR). ${ }^{3}$ In what follows, I overview applications of LCSR to hadronic form factors. I will also discuss a possibility to enlarge the region of accessible momentum transfers by employing the analyticity of the form factors.

*Talk at the Workshop "Shifmania, Crossing the boundaries: Gauge dynamics at strong coupling", May 14-17,2009, Minneapolis, USA 


\section{Hadronic form factors}

The simplest hadronic form factors parameterize electroweak transitions between two ground-state pseudoscalar mesons. A well-known example is the pion form factor generated by the quark electromagnetic current:

$$
\left\langle\pi(p+q)\left|j_{\mu}^{e m}\right| \pi(p)\right\rangle=(2 p+q)_{\mu} F_{\pi}\left(Q^{2}\right),
$$

where $j_{\mu}^{e m}=\frac{2}{3} \bar{u}(x) \gamma_{\mu} u(x)-\frac{1}{3} \bar{d}(x) \gamma_{\mu} d(x)$ and $Q^{2}=-q^{2}$. This form factor remains a popular test ground of QCD models, and is being used nowadays to probe the AdS/QCD approach (see, e.g., ${ }^{4,5}$ ).

An important constraint on any model of the pion form factor is the $Q^{2} \rightarrow \infty$ asymptotics ${ }^{6,7}$ :

$$
\left.F_{\pi}\left(Q^{2}\right)\right|_{Q^{2} \rightarrow \infty}=\frac{8 \pi \alpha_{s} f_{\pi}^{2}}{9 Q^{2}}\left|\int_{0}^{1} d u \frac{\varphi_{\pi}(u, \mu)}{1-u}\right|^{2},
$$

determined by the perturbative gluon exchange between the quark and antiquark constituents of the pion. In this factorization formula, the longdistance dynamics below the separation scale $\mu \sim \sqrt{Q^{2}}$ is encoded in the the pion distribution amplitude (DA) $\varphi_{\pi}(u, \mu)$. The latter is defined as the twist-2 part of the quark-antiquark vacuum-pion matrix element expanded near the light-cone:

$$
\left\langle\pi(p)\left|\bar{u}(x)[x, 0] \gamma_{\mu} \gamma_{5} d(0)\right| 0\right\rangle_{x^{2}=0}=-i p_{\mu} f_{\pi} \int_{0}^{1} d u e^{i u p \cdot x} \varphi_{\pi}(u, \mu),
$$

where $[x, 0]$ is the gauge factor and $\mu \sim 1 / \sqrt{\left|x^{2}\right|}$. The pion DA is a universal object, in a sense that it also enters factorization formulae for other pion form factors. The knowledge of $\varphi_{\pi}(u, \mu)$ is however not sufficient for a form factor calculation at finite $Q^{2}$. A major question remains to be addressed: how large are the soft contributions to the form factor (1) which start from $O\left(1 / Q^{4}\right)$.

Switching from the electromagnetic to the weak flavour-changing current $\bar{u} \gamma_{\mu} b$, one gets the $B \rightarrow \pi$ transition form factor:

$$
\left\langle\pi(p)\left|\bar{u} \gamma_{\mu} b\right| B(p+q)\right\rangle=(2 p+q)_{\mu} f_{B \pi}^{+}\left(q^{2}\right)+. .,
$$

where ellipses indicate the presence of the second form factor, due to nonconservation of the current. The $c \rightarrow d, s$ weak transitions generate similar $D \rightarrow \pi, K$ form factors. The role of large scale $Q^{2}$ is taken now by the heavy quark mass $m_{Q}(Q=b, c)$. In these heavy-light form factors there is again an interplay of hard and soft quark-gluon interactions. One can write 
an asymptotic factorization formula, similar to $(2)$, containing $\varphi_{\pi}(u, \mu)$ together with the heavy-meson DA. Again, such an ansatz is phenomenologically incomplete, because soft contributions have to be included. They play an even more important role in heavy-to-light form factors, since they are not power suppressed (in $1 / m_{Q}$ ) with respect to the hard factorizable part.

Heavy-light form factors are not only interesting hadronic objects. Their knowledge is of primary importance for flavour physics. For example, the $B \rightarrow \pi$ form factor is needed to extract the quark mixing parameter $\left|V_{u b}\right|$ from data on semileptonic decay $B \rightarrow \pi l \nu_{l}$, whereas $D \rightarrow \pi$ and $D \rightarrow$ $K$ form factors are used to determine $\left|V_{c d}\right|$ and, respectively, $\left|V_{c s}\right|$. The exploration of current and future experimental data on exclusive $B$ and $D$ decays demands accurate theoretical predictions for these form factors.

\section{QCD light-cone sum rules}

The method of LCSR was described in detail in many papers, (see, e.g. ${ }^{8-12}$ and the review ${ }^{13}$ ), hence I only give a very short outline. The central object is the correlation function of two quark currents between the vacuum and on-shell pion state. To give a familiar example, the calculation of the $B \rightarrow \pi$ form factors starts from the following expression:

$$
\int d^{4} x e^{i q x}\left\langle\pi(p)\left|T\left\{\bar{u}(x) \gamma_{\mu} b(x), \bar{b}(0) i m_{b} \gamma_{5} d(0)\right\}\right| 0\right\rangle=F\left(q^{2},(p+q)^{2}\right) p_{\mu}+\ldots,
$$

where only the invariant amplitude multiplying $p_{\mu}$ is relevant for the form factor $f_{B \pi}^{+}\left(q^{2}\right)$. At $q^{2},(p+q)^{2} \ll m_{b}^{2}$, the $b$-quark propagating in the correlation function has a large virtuality and the product of $b$-quark fields is expanded near the light-cone $x^{2} \sim 0$, leading to OPE for the correlation function, schematically:

$$
\begin{gathered}
F_{O P E}\left(q^{2},(p+q)^{2}\right)=\sum_{\Gamma_{a}} \int d^{4} x e^{i q x} C_{\left(\Gamma_{a}\right)}\left(x^{2}, m_{b}\right)\left\langle\pi(p)\left|\bar{u}(x) \Gamma_{a} d(0)\right| 0\right\rangle \\
+\sum_{\Gamma_{b}} \int d^{4} x e^{i q x} \int_{0}^{1} d v \tilde{C}_{\left(\Gamma_{b}\right)}^{\alpha \beta}\left(x^{2}, v, m_{b}\right)\left\langle\pi(p)\left|\bar{u}(x) G_{\alpha \beta}(v x) \Gamma_{b} d(0)\right| 0\right\rangle,
\end{gathered}
$$

where gauge factors are not shown, $\Gamma_{a, b}$ are various combinations of Dirac matrices. In the above, the short-distance coefficients $C_{\left(\Gamma_{a}\right)}$ and $\tilde{C}_{\left(\Gamma_{b}\right)}$ stemming from the $b$-quark propagator are calculated perturbatively, whereas the vacuum-pion matrix elements are expressed via pion light-cone DA's. The term with $\Gamma_{a}=\gamma_{\mu} \gamma_{5}$ yields the twist-2 DA defined in (3) and, in addition, the twist- 4 DA's. The terms with $\Gamma_{a}=i \gamma_{5}, \sigma_{\mu \nu} \gamma_{5}$ yield twist-3 DA's. 
Furthermore, the soft gluon emitted from the $b$-quark propagator enters quark-antiquark-gluon operators, shown in the second line in (6). These operators are decomposed in pion three-particle DA's of twist-3 and 4 . The dominant twist- 2 and twist- 3 contributions are calculated to $O\left(\alpha_{s}\right)$, obeying collinear factorization ${ }^{9-11}$. Subdominant twist-4 and three-particle contributions are power suppressed, justifying the truncated twist/Fock-state expansion. It is a future task to assess also the twist- 5 and 6 terms of this expansion.

Matching OPE and hadronic dispersion relation for the correlation function at $\left|(p+q)^{2}\right| \sim m_{b} \chi$, where $\chi$ is an intermediate scale $\gg \Lambda_{Q C D}$, one uses quark-hadron duality for the hadronic states above $B$ meson, obtaining:

$$
F_{O P E}\left(q^{2},(p+q)^{2}\right)=\frac{2 m_{B}^{2} f_{B} f_{B \pi}^{+}\left(q^{2}\right)}{m_{B}^{2}-(p+q)^{2}}+\int_{s_{0}^{B}}^{\infty} d s \frac{\left[\operatorname{Im} F\left(s, q^{2}\right)\right]_{O P E}}{s-(p+q)^{2}} .
$$

Substituting in (7) the amplitude $F_{O P E}$ calculated from (6), one obtains an approximate analytical answer for the form factor in terms of pion DA's convoluted with calculable coefficients. Inputs in this relation include the $b$ quark mass (in the $\overline{M S}$ scheme), $\alpha_{s}$, and the set of low-twist universal pion DA's. The decay constant $f_{B}$ is determined from two-point QCD (SVZ) sum rule. For other details, see, e.g. ${ }^{11}$.

Importantly, in the resulting LCSR for the form factor $f_{B \pi}^{+}$, the leading twist-2 and 3 terms start from $\alpha_{s}^{0}$, with NLO corrections being $O\left(\alpha_{s}\right)$ suppressed. Thus, in LCSR both soft (nonfactorizable) and hard (factorizable) contributions are taken into account and the soft one dominates. Importantly, higher twist contributions are suppressed by $\left(\Lambda_{Q C D} / m_{b}\right)$ and/or $\left(\Lambda_{Q C D} / \chi\right)$. Since the calculation is done at finite $m_{b}$, a transition from $b$ to $c$ quark in (5) is straightforward, yielding LCSR for $D \rightarrow \pi, K$ form factors. ${ }^{8,12,14,15}$

The universality of the method goes even further. Forming a vacuum $\rightarrow$ pion correlation function of the currents $j_{\mu}^{e m}$ and $\bar{u} \gamma_{\rho} \gamma_{5} d$, one obtains LCSR for the pion form factor $F_{\pi}\left(Q^{2}\right)^{16-18}$, valid at $Q^{2} \gg \Lambda_{Q C D}^{2}$. This sum rule predicts a substantial soft contribution at intermediate $Q^{2}$ and, simultaneously, reproduces the QCD asymptotics (2). Furthermore, employing vacuum $\rightarrow$ baryon correlation functions with a baryonic interpolating current and DA's, one is able to calculate various baryon form factors, e.g., the nucleon form factors ${ }^{19}$.

The method of LCSR has certain limitations. First of all, there is no "direct access" to the hadronic form factor. The analytical expression in a form of factorized OPE is obtained for the correlation function, whereas 
the form factor enters the pole term of the dispersion relation. One isolates it from the contributions of higher states to the dispersion relation, estimated using quark-hadron duality. This approximation introduces a sort of "systematic" uncertainty of the method. To keep it under control, one fits the threshold parameter $s_{0}^{B}$ in (7) by calculating the $B$-meson mass from the same LCSR. A typical accuracy of the form factors, calculated by this method is estimated at the level of $\sim \pm 15 \%$, by varying all input parameters and scales within their adopted interval. One of the main uncertainties is the shape of the twist-2 DA $\varphi_{\pi}(u)$ expressed via Gegenbauer moments.

The region of momentum transfer $q^{2}$ accessible for LCSR is restricted: $f_{B \pi}^{+}\left(q^{2}\right)$ is calculated at $q^{2} \ll\left(m_{B}-m_{\pi}\right)^{2}$, practically at $q^{2}<12-14 \mathrm{GeV}^{2}$, $f_{D \pi}^{+}\left(q^{2}\right)$ at $q^{2} \simeq 0$, and $F_{\pi}\left(Q^{2}\right)$ at $Q^{2} \geq 1 \mathrm{GeV}^{2}$. It is therefore an important task to access other regions of $q^{2}$ where more data are available. This is possible, provided one uses the analyticity of the form factors.

\section{Employing the analyticity}

Hadronic form factors are analytic functions of the momentum-transfer variable $q^{2}$. A typical dispersion relation which follows from the analyticity of the $B \rightarrow \pi$ form factor:

$$
f_{B \pi}^{+}\left(q^{2}\right)=\frac{m_{B^{*}} f_{B^{*}} g_{B^{*} B \pi}}{2\left(m_{B^{*}}^{2}-q^{2}\right)}+\frac{1}{\pi} \int_{\left(m_{B}+m_{\pi}\right)^{2}}^{\infty} d s \frac{\operatorname{Im} f_{B \pi}^{+}(s)}{s-q^{2}},
$$

takes into account the singularities located on the positive real $q^{2}$ axis, the lowest one being the ground-state $B^{*}$ pole (with the $B^{*} B \pi$ coupling defined as in $\left.{ }^{8}\right)$. Starting from the threshold at $q^{2}=\left(m_{B}+m_{\pi}\right)^{2}$, there are branch points and poles, generated by hadronic continuum states and excited resonances with $B^{*}$ quantum numbers. Note that, due to the QCD asymptotics $f_{B \pi}^{+}\left(q^{2} \rightarrow \infty\right) \sim 1 / q^{2}$ (similar to (2)), there are no subtractions in (8). Importantly, this dispersion relation is valid at any $q^{2}$. Hence, a practical way to enlarge the accessible $q^{2}$-region is to match the LCSR result to (8) at $q^{2} \ll m_{B}^{2}$ and analytically continue the dispersion relation. This however can only be done if a model/ansatz is introduced for the integral over the spectral density of higher states in (8), e.g., an effective pole ${ }^{20}$.

A less model-dependent approach employs conformal mapping (for earlier uses see ${ }^{21}$ ). One maps the complex $q^{2}$-plane where (8) is valid, onto the unit circle $|z|<1$ in the plane of the new variable: $z\left(q^{2}, t_{0}\right)=$ $\frac{\sqrt{t_{+}-q^{2}}-\sqrt{t_{+}-t_{0}}}{\sqrt{t_{+}-q^{2}}+\sqrt{t_{+}-t_{0}}}$, where $t_{+}=\left(m_{B}+m_{\pi}\right)^{2}$, and $t_{0}<t_{+}$is a parameter. 
There are many applications ${ }^{22}$ of this approach to $B \rightarrow \pi$ and other form factors, combined with perturbative QCD bounds obtained from the unitarity for the 2-point correlation function. In fact, the bounds are usually not restrictive, and, since the maximal values of $|z|$ corresponding to kinematical boundaries of $B \rightarrow \pi$ or $D \rightarrow \pi, K$ transitions are rather small, a simple Taylor series near $z=0$ suffices to parameterize the form factor. The last version of this series parameterization ${ }^{23}$ advocates a truncated power expansion:

$$
f_{B \pi}^{+}\left(q^{2}\right)=\frac{1}{1-q^{2} / m_{B^{*}}^{2}} \sum_{k=0}^{k_{\max }} a_{k}\left(z\left(q^{2}, t_{0}\right)\right)^{k},
$$

(with certain constraints on $a_{k}$ ), where the $B^{*}$-pole near the threshold is isolated. Three or four parameters are sufficient for a reasonably accurate parameterization. Employing (9), it is possible to go beyond the region where a LCSR calculation is valid. For that one has to fit the coefficients $a_{k}$ to the LCSR result for the form factor in the "trusted "region of $q^{2}$. After that, transforming $q^{2} \rightarrow z$, one continues (9) over $z$ beyond the initial region and finally transforms the variable $z$ back to $q^{2}$.

\section{Recent results for heavy-light form factors}

\section{1. $B \rightarrow \pi$ form factor and $\left|V_{u b}\right|$}

Let me first quote our update of the $B \rightarrow \pi$ form factor calculated from LCSR $^{11}$ with the result: $f_{B \pi}^{+}(0)=0.26_{-0.03}^{+0.04}$, where a recent very accurate determination of the $\overline{M S}$ b-quark mass ${ }^{24}$ was used. In this calculation no attempt yet was done to use the analytical continuation. On the contrary, in order to diminish the theoretical error for the form factor at $q^{2}=0$, the calculated form factor shape at $0<q^{2}<12 \mathrm{GeV}^{2}$ (the estimated region of validity of LCSR) was fitted to the $q^{2}$-distribution in $B \rightarrow \pi l \nu_{l}$, measured by BABAR collaboration. ${ }^{25}$ This fit allowed to tighten the constraints on the Gegenbauer moments of the twist-2 pion DA , yielding :

$$
\varphi_{\pi}(u, \mu)=6 u(1-u)\left(1+a_{2}(\mu) C_{2}^{3 / 2}(2 u-1)+a_{4}(\mu) C_{4}^{3 / 2}(2 u-1)\right),
$$

with $a_{2}(1 \mathrm{GeV})=0.16 \pm 0.01, a_{4}(1 \mathrm{GeV})=0.04 \pm 0.01\left(\right.$ neglecting $\left.a_{6}, \ldots\right)$. Note that these intervals are quite narrow and are within broader "world averages" (see e.g. ${ }^{26}$ ). Deviation of the DA from its asymptotic form is thus rather mild. Finally, in ${ }^{11}$ the form factor at $q^{2}=0$ was used to extract $\left|V_{u b}\right|$ from the data on $B \rightarrow \pi l \bar{\nu}$. This and other recent $\left|V_{u b}\right|$ determinations from $B \rightarrow \pi l \nu_{l}$ are summarized in the following table: 


\begin{tabular}{|c|c|c|c|}
\hline [Ref. $]$ & $\begin{array}{c}f_{B \pi}^{+}\left(q^{2}\right) \\
\text { calculation }\end{array}$ & $\begin{array}{c}f_{B \pi}^{+}\left(q^{2}\right) \\
\text { input }\end{array}$ & $\left|V_{u b}\right| \times 10^{3}$ \\
\hline 27 & lattice & - & $3.78 \pm 0.25 \pm 0.52$ \\
\hline${ }^{28}$ & lattice & - & $3.55 \pm 0.25 \pm 0.50$ \\
\hline${ }^{10}$ & LCSR & - & $3.5 \pm 0.4 \pm 0.1$ \\
\hline 29 & - & lattice $\oplus$ LCSR & $3.47 \pm 0.29 \pm 0.03$ \\
\hline${ }^{11}$ & LCSR & - & $3.5 \pm 0.4 \pm 0.2 \pm 0.1$ \\
\hline${ }^{23}$ & - & lattice $\oplus$ LCSR & $3.54 \pm 0.24$ \\
\hline
\end{tabular}

Importantly, both LCSR and lattice QCD determinations are in a good agreement with $\left|V_{u b}\right|=\left(3.5_{-0.14}^{+0.15}\right) \times 10^{-3}$ inferred from the CKM unitarity triangle fits ${ }^{30}$. Let me also mention a LCSR calculation ${ }^{31}$ of $B \rightarrow K$ and $B_{s} \rightarrow K$ form factors (needed e.g., for models of rare exclusive $B$ decays).

\section{2. $D \rightarrow \pi, K$ form factors and $\left|V_{c d}\right|,\left|V_{c s}\right|$}

Following the same calculational scheme as for $B \rightarrow \pi^{12}$ and $B \rightarrow K^{31}$ transitions, using the same set of pion and kaon DA's and $\overline{M S}$ c-quark mass from $^{24}$, we calculated ${ }^{12}$ the $D \rightarrow \pi$ and $D \rightarrow K$ form factors. In the latter, the $S U(3)_{f l}$ violation is taken into account in $O\left(m_{s}\right)$. Predictions for these form factors from lattice QCD and LCSR are presented in this table:

\begin{tabular}{|cc|c|c|}
\hline Method & {$[$ Ref.] } & $f_{D \pi}^{+}(0)$ & $f_{D K}^{+}(0)$ \\
\hline Lattice QCD & 33 & $0.57 \pm 0.06 \pm 0.02$ & $0.66 \pm 0.04 \pm 0.01$ \\
& 34 & $0.64 \pm 0.03 \pm 0.06$ & $0.73 \pm 0.03 \pm 0.07$ \\
& 35 & $0.74 \pm 0.06 \pm 0.04$ & $0.78 \pm 0.05 \pm 0.04$ \\
\hline LCSR & 14 & $0.65 \pm 0.11$ & $0.78_{-0.15}^{+0.2}$ \\
& 15 & $0.63 \pm 0.11$ & $0.75 \pm 0.12$ \\
& 12 & $0.67_{-0.07}^{+0.10}$ & $0.75_{-0.08}^{+0.11}$ \\
\hline
\end{tabular}

Our calculation was then used to determine $\left|V_{c d}\right|$ and $\left|V_{c s}\right|$ with an improved accuracy from the recent CLEO data ${ }^{32}$ on semileptonic $D$ decays. (see ${ }^{12}$ for more details and numerical results).

In addition, the series parameterization similar to (9) was used to enlarge the accessible $q^{2}$ region. The form factors were calculated at $q^{2}<0$ (still within the region of validity of LCSR), and fitted to the $z$ parameterization. The result was then analytically continued, to cover the whole kinematical region $0 \leq q^{2} \leq\left(m_{D}-m_{\pi(K)}\right)^{2}$ of $D \rightarrow \pi(K) l \nu_{l}$ decay. The predicted shape of $f_{D \pi}^{+}\left(q^{2}\right)$ normalized at $q^{2}=0$ is compared with the one measured by CLEO ${ }^{32}$ in Fig. 1. 


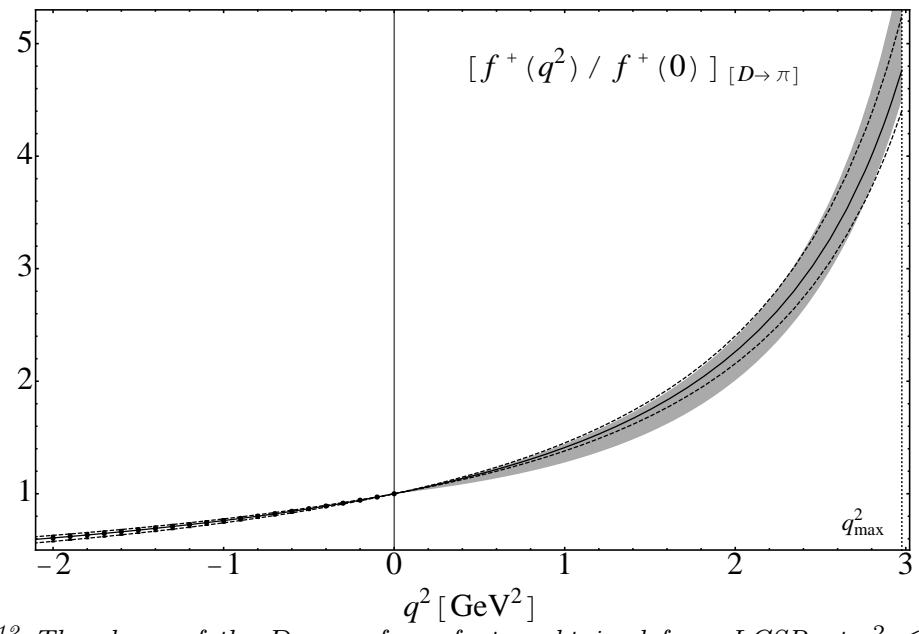

Fig. 1. ${ }^{12}$ The shape of the $D \rightarrow \pi$ form factor obtained from LCSR at $q^{2} \leq 0$, fitted to series parameterization (solid line and dashed lines indicating uncertainties), and compared with the shape measured by $C L E O^{32}$ at $0 \leq q^{2} \leq\left(m_{D}-m_{\pi}\right)^{2}$ (shaded region).

\section{Pion form factor}

As already mentioned, the LCSR approach allows one to calculate any hadronic form factor, provided the momentum transfer is sufficiently below the hadronic threshold in the channel of the transition current. It is therefore interesting to return to the pion e.m. form factor $F_{\pi}\left(Q^{2}\right)$ which is quite sensitive to the pion twist-2 DA and recalculate it from LCSR ${ }^{17,18}$. In this sum rule the twist- 2 term with $O\left(\alpha_{s}\right)$ corrections and twist-4,6 terms are taken into account. With the pion DA given by (10) and taking the remaining input from ${ }^{18}$, I recalculated the pion e.m. form factor at $Q^{2}=1.0-5.0$ $\mathrm{GeV}^{2}$. The result is presented in Fig. 2, for the central values of the input. Conservatively, a $\pm 15 \%$ uncertainty still has to be added. The agreement with the recent accurate data at $Q^{2} \leq 2.45 \mathrm{GeV}^{2}$, obtained at Jefferson $\mathrm{Lab}^{36}$ is encouraging.

Furthermore, it is easy to write down an analog of series parameterization (9) for $F_{\pi}\left(Q^{2}\right)$, which is simply a polynomial in $z$ in this case (the lowest $\rho$-meson pole is far above the threshold) and continue the form factor to small $Q^{2}$. The result shown in Fig. 2 is consistent with the direct measurement of the pion form factor in this region ${ }^{37}$ and correctly reproduces $\lim _{Q^{2} \rightarrow 0} F_{\pi}\left(Q^{2}\right)=1$ (within the accuracy of the sum rule).

I conclude that the pion DA (10) used in calculating the heavy-light form factors successfully passes the pion form factor test. A more detailed 


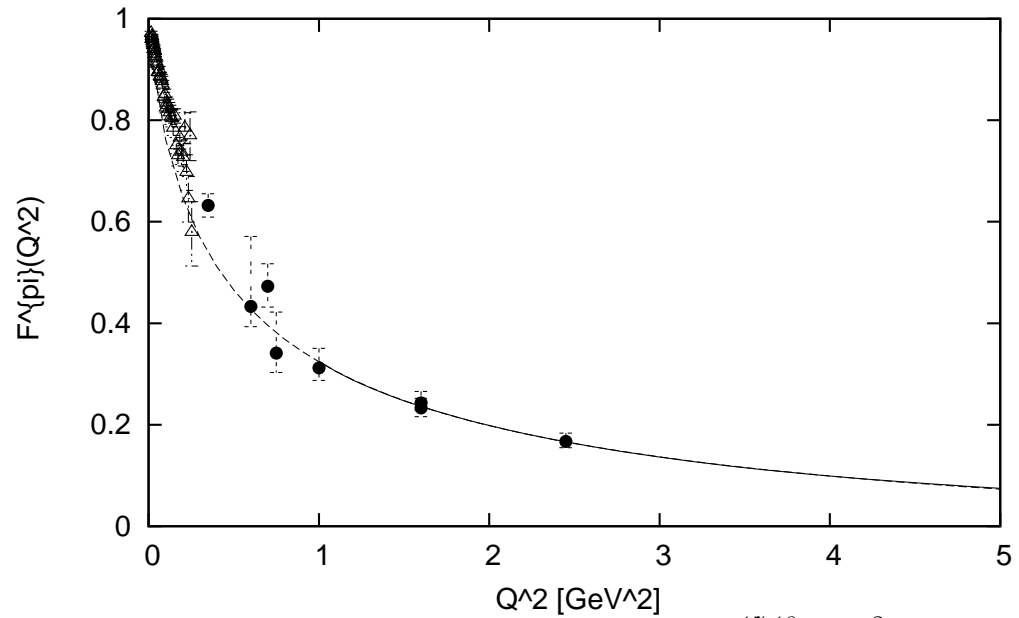

Fig. 2. The pion e.m. form factor calculated from LCSR ${ }^{17,18}$ at $Q^{2}=1-5 \mathrm{GeV}^{2}$ (solid), fitted to series parameterization and analytically continued to smaller $Q^{2}$ (dashed), compared with JLAB data ${ }^{36}$ (filled points) and $N A 7$ data ${ }^{37}$ (open triangles)

study will be published elsewhere.

\section{Photon-pion transition form factor}

(a comment added after the talk)

The pion twist-2 DA also determines the QCD asymptotics ${ }^{7,38}$ of the $\gamma^{*} \gamma^{*} \rightarrow \pi^{0}$ amplitude:

$$
F^{\gamma^{*} \pi}\left(Q^{2}, k^{2}\right)=\frac{\sqrt{2} f_{\pi}}{3} \int_{0}^{1} d u \frac{\varphi_{\pi}(u, \mu)}{Q^{2}(1-u)+\left|k^{2}\right| u} \oplus O\left(\alpha_{s}\right),
$$

where both photon virtualities, $Q^{2}=-q^{2}$ and $\left|k^{2}\right| \neq Q^{2}$, are sufficiently large. The $\gamma^{*} \gamma^{*} \rightarrow \pi^{0}$ amplitude is however only measured ${ }^{44-46}$ when one of the photons is almost real $\left(k^{2} \simeq 0\right)$, in which case it reduces to the photonpion transition form factor $F^{\gamma \pi}\left(Q^{2}\right)=F^{\gamma^{*} \pi}\left(Q^{2}, 0\right)$. Note that at $k^{2}=0$ the factorization formula (11) is incomplete, because the real photon has a long-distance, hadronlike component. Hence, substituting a certain model of $\varphi_{\pi}(u, \mu)$ in (11) and comparing the result at $k^{2}=0$ with experimental data on $F^{\gamma \pi}\left(Q^{2}\right)$, is not yet a conclusive test of QCD. Calculations which take into account long-distance component of the photon can be found in the literature, (see, e.g. ${ }^{39}$ ). 


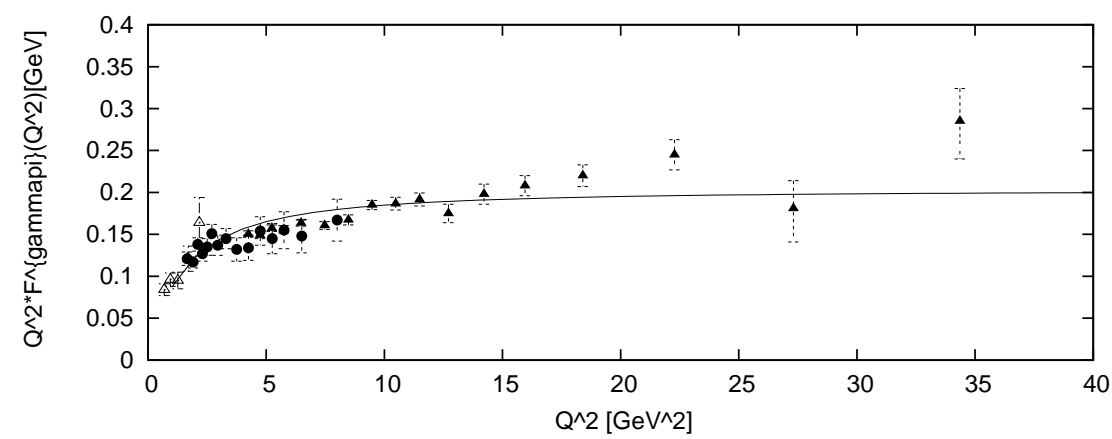

Fig. 3. Photon-pion form factor $Q^{2} F^{\gamma \pi}\left(Q^{2}\right)$ calculated from a combination of LCSR and dispersion relation ${ }^{40}$, including $O\left(\alpha_{s}\right)$ corrections ${ }^{41}$, compared with the CELLO 44 (open triangles), $C L E O^{45}$ (full points) and BABAR ${ }^{46}$ data (full triangles)

A method to calculate $F^{\gamma \pi}\left(Q^{2}\right)$, combining LCSR with the analytical continuation was suggested in ${ }^{40}$. The $\gamma^{*} \gamma^{*} \rightarrow \pi^{0}$ amplitude was calculated using light-cone OPE, with both $Q^{2}=-q^{2}$ and $\left|k^{2}\right|$ large, adding also the twist-4 term to (11). (A calculation of the next, twist-6 term remains a future task.) Equating this amplitude to the dispersion relation in $k^{2}$ :

$$
F_{O P E}^{\gamma^{*} \pi}\left(Q^{2}, k^{2}\right)=\frac{\sqrt{2} f_{\rho} F_{\rho \pi}\left(Q^{2}\right)}{m_{\rho}^{2}-k^{2}}+\frac{1}{\pi} \int_{s_{0}}^{\infty} \frac{\operatorname{Im} F^{\gamma^{*} \pi}\left(Q^{2}, s\right)}{s-k^{2}},
$$

where $\rho$ and $\omega$ contributions are combined in one resonance term with $m_{\rho} \simeq m_{\omega}$, one calculates the $\rho \rightarrow \pi$ form factor using the technique of LCSR and employs quark-hadron duality for the integral above the threshold $s_{0}$. After that a smooth transition to $k^{2} \rightarrow 0$ in the dispersion relation is possible, yielding the form factor $F^{\gamma \pi}\left(Q^{2}\right)$. The $O\left(\alpha_{s}\right)$ corrections in this approach were calculated in ${ }^{41}$, and some recent updates can be found in 42,43 .

Intrigued by the new BABAR data ${ }^{46}$, I returned to the calculation of $F^{\gamma \pi}\left(Q^{2}\right)$ from (12), using the twist-2 pion DA (10), the remaining input from ${ }^{40}$, and adding the $O\left(\alpha_{s}\right)$ corrections from ${ }^{41}$. The result is shown in Fig. 3 (for the central values of the input). At large $Q^{2}$, as expected, $Q^{2} F^{\gamma \pi}\left(Q^{2}\right)$ tends to a constant ${ }^{\text {a }}$. In spite of a reasonable agreement with the old CELLO and CLEO data at low $Q^{2}$ and with the new BABAR data up to $q^{2} \leq 15 \mathrm{GeV}^{2}$, this calculation (as well as the one $\mathrm{in}^{43}$ ) does not

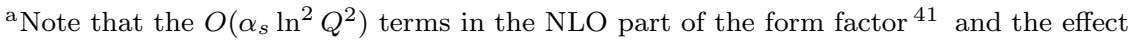
of their resummation still have to be assessed. 
reproduce the tendency of the increasing $Q^{2} F^{\gamma \pi}\left(Q^{2}\right)$ at larger $Q^{2}$ visible in the data, although the experimental errors in this region are rather large. An additional measurement, e.g., by Belle collaboration is important to confirm these very interesting data.

\section{Acknowledgments}

I am very grateful to FTPI for inviting me to take part in the celebration of Misha Shifman's extraordinary achievements in particle theory. Due to his important and enlightening contributions, deep connections between QCD and hadron phenomenology were found and are being extensively used.

I acknowledge a useful discussion with Vladimir Braun. This work is supported by the Deutsche Forschungsgemeinschaft under the contract No. KH205/1-2.

\section{References}

1. M. Shifman, Persistent challenges of quantum chromodynamics, Lilienfeld Prize Lecture, Int. J. Mod. Phys. A 21 (2006) 5695 [arXiv:hep-th/0606015].

2. M. A. Shifman, A. I. Vainshtein and V. I. Zakharov, Nucl. Phys. B 147, 385, 448 (1979).

3. I.I. Balitsky, V.M. Braun, A.V. Kolesnichenko, Nucl. Phys. B312 (1989) 509; V. M. Braun and I. E. Filyanov, Z. Phys. C44 (1989) 157;

V. L. Chernyak and I. R. Zhitnitsky, Nucl. Phys. B345 (1990) 137.

4. A. Radyushkin, arXiv:0907.4585 [hep-ph], talk at this workshop.

5. J. Erlich, arXiv:0908.0312 [hep-ph], talk at this workshop.

6. V. L. Chernyak and A. R. Zhitnitsky, JETP Lett. 25 (1977) 510;

A. V. Efremov and A. V. Radyushkin, Phys. Lett. B 94, 245 (1980).

7. G. P. Lepage and S. J. Brodsky, Phys. Lett. B 87, 359 (1979); Phys. Rev. D 22, 2157 (1980).

8. V. M. Belyaev, V. M. Braun, A. Khodjamirian and R. Rückl, Phys. Rev. D 51 (1995) 6177.

9. A. Khodjamirian, R. Rückl, S. Weinzierl and O. I. Yakovlev, Phys. Lett. B 410 (1997) 275;

E. Bagan, P. Ball and V. M. Braun, Phys. Lett. B 417, 154 (1998).

10. P. Ball and R. Zwicky, Phys. Rev. D 71 (2005) 014015.

11. G. Duplancic, A. Khodjamirian, T. Mannel, B. Melic and N. Offen, JHEP 0804, 014 (2008).

12. A. Khodjamirian, C. Klein, T. Mannel, N. Offen, arXiv:0907.2842 [hep-ph].

13. P. Colangelo and A. Khodjamirian, In Shifman, M. (ed.): At the frontier of particle physics/Handbook of QCD, vol. 3, 1495-1576; hep-ph/0010175.

14. A. Khodjamirian, R. Ruckl, S. Weinzierl, C. W. Winhart and O. I. Yakovlev, Phys. Rev. D 62 (2000) 114002.

15. P. Ball, Phys. Lett. B 641 (2006) 50.

16. V. M. Braun and I. E. Halperin, Phys. Lett. B 328, 457 (1994). 
17. V. M. Braun, A. Khodjamirian and M. Maul, Phys. Rev. D 61 (2000) 073004.

18. J. Bijnens and A. Khodjamirian, Eur. Phys. J. C 26, 67 (2002).

19. V. M. Braun, arXiv:hep-ph/0608231, in Minneapolis 2006, Continuous advances in $\mathrm{QCD}^{*}$, pp 42-57;

V. M. Braun, A. Lenz and M. Wittmann, Phys. Rev. D 73 (2006) 094019.

20. D. Becirevic and A. B. Kaidalov, Phys. Lett. B 478 (2000) 417.

21. N. N. Meiman, Sov. Phys. JETP 17 (1963) 830;

B. V. Geshkenbein, B. L. Ioffe, Sov. Phys. JETP 17 (1963) 820;

S. Okubo, Phys. Rev. D 4, 725 (1971);

C. Bourrely, B. Machet and E. de Rafael, Nucl. Phys. B 189, 157 (1981).

22. C. G. Boyd, B. Grinstein and R. F. Lebed, Phys. Rev. Lett. 74, 4603 (1995); L. Lellouch, Nucl. Phys. B 479, 353 (1996);

M. C. Arnesen, B. Grinstein, I. Z. Rothstein and I. W. Stewart, Phys. Rev. Lett. 95 (2005) 071802;

T. Becher and R. J. Hill, Phys. Lett. B 633, 61 (2006).

23. C. Bourrely, I. Caprini and L. Lellouch, Phys. Rev. D 79 (2009) 013008.

24. J. H. Kuhn, M. Steinhauser and C. Sturm, Nucl. Phys. B 778 (2007) 192;

R. Boughezal, M. Czakon and T. Schutzmeier, Phys. Rev. D 74, 074006 (2006).

25. B. Aubert et al. [BABAR Collaboration], Phys. Rev. Lett. 98 (2007) 091801.

26. P. Ball, V. M. Braun and A. Lenz, JHEP 0708 (2007) 090.

27. M. Okamoto, PoS LAT2005 (2006) 013 [arXiv:hep-lat/0510113].

28. E. Dalgic et al. , Phys. Rev. D 73, 074502 (2006) [Erratum-ibid. D 75, 119906 (2007)].

29. J. M. Flynn and J. Nieves, Phys. Rev. D 76, 031302 (2007).

30. J. Charles et al. [CKMfitter Group], Eur. Phys. J. C 41 (2005) 1, Moriond 2009 update at http://ckmfitter.in2p3.fr .

31. G. Duplancic and B. Melic, Phys. Rev. D 78, 054015 (2008).

32. D. Besson [CLEO Collaboration], arXiv:0906.2983 [hep-ex].

33. A. Abada et al. Nucl. Phys. B 619, 565 (2001).

34. C. Aubin et al. [Fermilab Lattice Collaboration, MILC Collaboration and HPQCD Collaboration], Phys. Rev. Lett. 94, 011601 (2005).

35. A. Al-Haydari et al. [QCDSF Collaboration], arXiv:0903.1664 [hep-lat].

36. G. M. Huber et al. [Jefferson Lab Collaboration], Phys. Rev. C 78 (2008) 045203.

37. S. R. Amendolia et al. [NA7 Collaboration], Nucl. Phys. B 277 (1986) 168.

38. S. J. Brodsky and G. P. Lepage, Phys. Rev. D 24, 1808 (1981).

39. A. V. Radyushkin and R. T. Ruskov, Nucl. Phys. B 481, 625 (1996).

40. A. Khodjamirian, Eur. Phys. J. C 6 (1999) 477.

41. A. Schmedding and O. I. Yakovlev, Phys. Rev. D 62, 116002 (2000).

42. S. S. Agaev, Phys. Rev. D 72, 114010 (2005) [Erratum-ibid. D 73, 059902 (2006)].

43. S. V. Mikhailov and N. G. Stefanis, Nucl. Phys. B 821, 291 (2009).

44. H. J. Behrend et al. [CELLO Collaboration], Z. Phys. C 49, 401 (1991).

45. J. Gronberg et al. [CLEO Collaboration], Phys. Rev. D 57 (1998) 33.

46. B. Aubert [The BABAR Collaboration], arXiv:0905.4778 [hep-ex]. 